\title{
Climate Change Alters Diffusion of Forest Pest: A Model Study
}

\author{
Woo Seong Jo and Beom Jun Kim $*$ \\ Department of Physics, Sungkyunkwan University, Suwon 16419, Republic of Korea \\ Hwang-Yong Kim \\ International Technology Cooperation Center, Rural Development Administration, Jeonju 54875, Republic of Korea
}

\begin{abstract}
Population dynamics with spatial information is applied to understand the spread of pests. We introduce a model describing how pests spread in discrete space. The number of pest descendants at each site is controlled by local information such as temperature, precipitation, and the density of pine trees. Our simulation leads to a pest spreading pattern comparable to the real data for pine needle gall midge in the past. We also simulate the model in two different climate conditions based on two different representative concentration pathways scenarios for the future. We observe that after an initial stage of a slow spread of pests, a sudden change in the spreading speed occurs, which is soon followed by a large-scale outbreak. We found that a future climate change causes the outbreak point to occur earlier and that the detailed spatio-temporal pattern of the spread depends on the source position from which the initial pest infection starts.
\end{abstract}

PACS numbers: 89.75.-k, 87.23.Cc, 87.53.Vb

Keywords: Population dynamics, Pest spreading, Lattice model, Climate change, RCP scenarios

\section{INTRODUCTION}

Population dynamics has been established as one of the successful mathematical methods for describing the temporal dynamics of populations in physics $[1] 3]$ and biology [4-6]. The methodology in population dynamics often takes different forms: Full-mixing models (i.e., meanfield models in physics) with quantities averaged over whole spatial locations, and models with spatial information explicitly taken into account (i.e., structured population models) have been widely used. The advantage of the mean-field approach is that the equation for population dynamics often becomes mathematically tractable, and one can clearly understand what happens in a longtime limit, i.e., species will be extinct or not. However, such a mathematical tractability comes at a cost: In the real world of nature, all species are locally embedded in a large-scale geographic space with finite dimensions of two or three. Living agents located in space cannot interact with all other agents in a finite time, and their behaviors are spatially and temporarily limited. Such a spatial constraint leads to remarkably different results, and the solution from the mean-field approach often fails to explain empirical observations. For this reason, researchers have been trying to integrate spatial information into dynamics in the design of their models to mimic the real world of nature.

Cellular Automata (CA) is often used as a tool for spatially explicit models. In the CA approach, space is approximated as a discrete lattice whose resolution needs to be fine enough to properly describe local information. Besides discretized space, the quantities describing the system are assigned to each of the sites. It is one of differences between the CA and the individual-based model,

\footnotetext{
* Corresponding author, E-mail: beomjun@skku.edu
}

where agents have their own properties regardless of their location. In the present work, we use a discrete lattice like CA, but use the population density as a local variable defined on each lattice point. The time evolution of the population density is given by a dynamic equation with a transition rate and local interactions as key ingredients similarly to Ref. 7. A model defined in a discrete lattice is very efficient from a the computational point of view because much spatial information can be integrated as spatially discrete variables. Such approaches have been utilized for various biological systems like vegetation dynamics [8 10], epidemics [11, 12], and spread of pests [13 15], as well as variant subjects in physics [1618].

The spread of pests in vegetation has been a critical issue because a very long time is needed for ruined vegetation to recover. In Japan and South Korea, the spread of the pine needle gall midge (PNGM) has been a serious problem from the early 1900's. When a pine tree is parasitized by PNGMs, the infected trees wither and can eventually die. Ecological studies of the PNGM have been conducted to identify conditions for the PNGM to spread broadly, and the spreading pattern of withered forest have also been empirically investigated [19 21]. Computational approaches with more available data have been developed, and it has become possible to use simulational methods for the study of PNGM spread [15]. A machine-learning technique has been used to forecast the spread of damage 22, 23], and images from satellites have also been analyzed 24]. Although population dynamics in a discrete lattice can be a very useful research framework due to its flexibility and computational efficiency, not many studies, with a few exception, have used this method 15.

Accurate prediction of future climate change is very difficult. Even when the computational framework for the forecast is given, the forecast results can differ de- 
pending on the assumptions the model uses. In reality, the number of quantitative assumptions for future environmental conditions can be huge. If different studies use different assumptions among many possibilities, comparing the results of one study with those of other studies is difficult. Scholars in the research area of future climate change have agreed on a few official scenarios. The first scenario was introduced by the Intergovernmental Panel on Climate Change (IPCC) in 1992 25]. In 2000, the IPCC published the second version of a scenario called the Special Report on Emissions Scenarios (SRES) 26]. The SRES have four categories, which were discussed in two follow-up reports, i.e., Third Assessment Report (TAR) and Assessment Report Four (AR4). Each category shows the amount of emission of greenhouse gas, which is determined by the speed of development, human population, and other possible causes for generating gas. The latest scenario of climate change is called Representative Concentration Pathways (RCPs) [27], which is an improved version of the SRES with the latest climate data in 2014. RCPs contain four categories called RCP2.6, RCP4.5, RCP6.0, and RCP8.5. The first category, RCP2.6 assumes that the greenhouse effect can be reduced by nature itself, which is doubtful right now. As the number in each category increases from 2.6 to 8.5, the emission of greenhouse gas is assumed to increase more in the future. The RCP8.5 scenario assumes that the concentration of greenhouse gas will follow the current trend. Most studies on climate change use one of the RCP scenarios with different greenhouse gas concentrations for the future and try to predict local climate variables such as temperature and precipitation.

In this paper, we introduce a model to describe the temporal dynamics and the spread of a population of PNGMs in the two-dimensional lattice model of South Korea. For consistency with real field data, small initial PNGM densities are assigned to three cities, Incheon, Mokpo and Busan, and the spread patterns are predicted by using climate scenarios RCP 4.5 and RCP 8.5 . We observe that the patterns of spread are comparable with the field data observed in the past. Furthermore, we also investigate how different climate scenarios affect the future prediction of the spread pattern of PNGM.

\section{METHOD}

\section{Climate Conditions}

Future climate changes in South Korea have been estimated based on the RCP scenarios. Each such estimate, RegCM4 [28], SNURCM [29], GRIMs [30], WRF 31], and HadGEM3-RA [32], has limitations of its own and contains inevitable uncertainty about initial conditions. Later, MME5s [33], which uses the concept of ensemble, combining estimates from five climate predictions, was suggested in order to overcome such limitations. In the present study, we use the future climate estimates of the MME5s in Climate Information Portal [34]. The climate data from MME5s have a fine spatial resolution of $1 \mathrm{~km} \times 1 \mathrm{~km}$ for every month from 2021 to 2050 . We choose climate data based on two RCP scenarios, RCP 4.5 and RCP8.5, to compare our results based on different assumptions for the future concentration of greenhouse gas.

\section{Model}

Once the spatial climate conditions are fixed from the data based on MME5s with RCP4.5 or RCP8.5, we then apply our structured CA model for the spread of the PNGM. Heretofore, we will simply call a PNGM as a midge. Let $\rho_{t}(\mathbf{r})$ be the density of adult midges at a discrete lattice point $\mathbf{r}$ and at a discrete time $t$ (we fix the unit of time as unity, which corresponds to one year, the life cycle of the PNGM). The adult midge density is modeled to evolve in time as

$$
\rho_{t}(\mathbf{r})=\lambda(\mathbf{r} ; T, P, \psi) \bar{\rho}_{t}(\mathbf{r}) \exp \left[-\frac{\bar{\rho}_{t}(\mathbf{r})}{\psi_{t}(\mathbf{r})}\right],
$$

where $\bar{\rho}_{t}(\mathbf{r})$ is the density of midge eggs to be explained below and $\lambda(\mathbf{r})$ is the position-dependent survival rate, which depends on the local values of the temperature $T$, the precipitation $P$, and the tree density $\psi$. The previous generation of adult midges at $t-1$ cannot survive more than a year; thus, the adult midges at $t$ are all born from midge eggs left by adult midges in the previous generation at $t-1$. Accordingly, the midge density $\rho_{t}$ at $t$ does not directly depend on $\rho_{t-1}$, but it depends on the density $\bar{\rho}_{t}$ of midge eggs left by the previous generation of adult midges at time $t-1$. When $\bar{\rho}_{t}(\mathbf{r}) / \psi_{t}(\mathbf{r})$ is small, $\exp \left[-\bar{\rho}_{t}(\mathbf{r}) / \psi_{t}(\mathbf{r})\right] \approx 1-\bar{\rho}_{t}(\mathbf{r}) / \psi_{t}(\mathbf{r})$, and the right-hand side of Eq. (11) takes the form of the corresponding term in the standard logistic equation. In the original logistic equation, the population growth is controlled by the carrying capacity. The population can exceed the carrying capacity in the logistic equation, but then the growth rate becomes negative, reducing the population afterward. However, our growth model governed by Eq. (10) is somewhat different. If we replace the exponential term by the standard form in the original logistic equation, the midge density can be negative, causing our growth model to fail. In order to avoid this catastrophe in evolving dynamics, we have, thus, introduced the form $x e^{-x}$, instead of the quadratic form in the conventional logistic equation. The exponential form is equivalent to the standard logistic form $x(1-x)$ when $x$ becomes smalls and prevents the midge density from being negative when the egg density exceeds the tree density. Therefore, the advantage of our growth model (11) is twofold: it is consistent with the conventional logistic equation when the egg density is small, and it also controls the unwanted catastrophic failure in the model. One can recognize then that the tree density $\psi$ plays the role of the carrying capacity of 
midge eggs, which appears to be a reasonable approximation since adult midges lay eggs in pine trees. From this reasoning, one can see that we normalize the egg density $\bar{\rho}$ with respect to the tree density $\psi$ in such a way that the maximum egg density is defined to be proportional to the tree density. The proportionality constant can be absorbed into the definition of the growth rate $\lambda$, giving us Eq. (1).

The position-dependent survival rate $\lambda(\mathbf{r})$ in Eq. (1) is affected by local climate conditions like the temperature and the precipitation. In our model, one unit of time corresponds to one year; thus, the temperature $T$ and the precipitation $P$ need to be defined in some average sense for each year. We note that most midges become adults from pupa and lay eggs in June; thus, we use the timeaveraged temperature in June as $T$. In existing literature on the PNGM 22], the moisture of soil has been shown to be one of the crucial factors for a midge to grow into an adult. We, thus, calculate the average precipitation from March to May and use it for $P$. The survival of the midge larvae to become an adult midge is known to depend on strongly the temperature [20]: Survival probability shows a slightly skewed bell shape for the temperature range between $12^{\circ} \mathrm{C}$ and $30^{\circ} \mathrm{C}$. We thus use such suitable climate conditions for the PNGM to grow to adults and write the survival rate $\lambda(\mathbf{r})$ as

$$
\lambda(\mathbf{r} ; T, P, \psi)=A \psi_{t}(\mathbf{r})\left[T_{t}(\mathbf{r})-T_{\min }\right]\left[T_{\max }-T_{t}(\mathbf{r})\right] G\left[P_{t}(\mathbf{r})\right],
$$

where $A$ is the normalization constant to make $\lambda$ in the interval $[0,1]$ and $P(\mathbf{r})$ is the above mentioned time-averaged precipitation at $\mathbf{r}$. To mimic the suitable temperature range, we have chosen in Eq. (22) a concave quadratic form to approximate the bell-shaped curve reported in Ref. 20 with $T_{\min }=12^{\circ} \mathrm{C}$ and $T_{\max }=30^{\circ} \mathrm{C}$. Although more rain has been reported to be better in Ref. 22, we suppose that the marginal gain must be very small if the precipitation is too large. As a rough approximation for such a dependence on $P$, we write

$$
G\left[P_{t}(\mathbf{r})\right]= \begin{cases}0, & \text { for } P_{t}(\mathbf{r})<P_{\min }, \\ {\left[P_{t}(\mathbf{r})-P_{\min }\right] /\left(P_{\max }-P_{\min }\right),} & \text { for } P_{\min } \leq P_{t}(\mathbf{r}) \leq P_{\max } \\ 1, & \text { for } P_{t}(\mathbf{r})>P_{\max },\end{cases}
$$

where $P_{\min }=20 \mathrm{~mm}$ and $P_{\max }=100 \mathrm{~mm}$ are suitably chosen based on the average precipitation from March to May in Gyeonggi province in Korea. Since $G(P)$ has a value between 0 and 1 , the normalization constant $A$ in Eq. (2) is written as $4 /\left(T_{\max }-T_{\min }\right)^{2}$ to make the maximum survival rate unity.

Once Eq. (1) combined with Eqs. (2) and (3) yields the midge density at every site at time $t$, we need to describe how adult midges lay eggs in space. A plausible assumption is that adult midges at $\mathbf{r}$ lay eggs on trees located not far from $\mathbf{r}$. Accordingly, the heterogeneity of the tree density must be taken into account for the spread pattern of eggs. In our notation, adult midges at time $t$ are from eggs at time $t$ which were laid by adult midges at $t-1$. Accordingly, the egg density $\bar{\rho}_{t}$ at time $t$ must be related to midge density $\rho_{t-1}$ at time $t-1$, and we write the relation in the form:

$$
\bar{\rho}_{t}(\mathbf{r})=\sum_{\mathbf{r}^{\prime} \in \mathcal{N}(\mathbf{r})} \rho_{t-1}\left(\mathbf{r}^{\prime}\right) \omega_{t-1}\left(\mathbf{r}^{\prime}, \mathbf{r}\right),
$$

where $\mathcal{N}(\mathbf{r})$ is the set of discrete lattice points within a distance of $5 \mathrm{~km}$ from $\mathbf{r}$ since the speed of midge spread has been reported to be about $5 \sim 6 \mathrm{~km} /$ year 35$]$. In Eq. (4), $\omega_{t-1}\left(\mathbf{r}^{\prime}, \mathbf{r}\right)$ controls how many eggs are laid at $\mathbf{r}$ by adult midges at $\mathbf{r}^{\prime}$, and we assume the following form:

$$
\omega_{t}\left(\mathbf{r}^{\prime}, \mathbf{r}\right)=g_{m} \frac{\psi_{t}(\mathbf{r})}{\sum_{\mathbf{r}^{\prime \prime} \in \mathcal{N}\left(\mathbf{r}^{\prime}\right)} \psi_{t}\left(\mathbf{r}^{\prime \prime}\right)}
$$

which implies that the adult midges at $\mathbf{r}^{\prime}$ move into their local neighbors and lay eggs in proportion to the local tree density. In this process, we assume that all midges have the same reproductive capability; thus, the growth parameter of a midge, $g_{m}$ in Eq. (5), is suitably set to a uniform value of 5.0 in our simulations.

Various pest species including PNGMs need host vegetation for reproduction. Once invaded by parasites, hosts become weak due to the lack of water and nutrients and can face fatal situations in harsh circumstances. The pine tree is the host vegetation for PNGMs, and once infected by PNGMs it loses leaves. Our equations, Eqs. (10)-(5), so far deal with how the midge density and egg density evolve in time. The last ingredient in our model is to mimic the effect of midges on pine trees. In the absence of midges, the tree density increases at a constant rate every year until the maximum possible density is approached. On the other hand, a large midge density reduces the tree density. The density $\psi_{t}(\mathbf{r})$ of pine trees is thus assumed to evolve in time as

$$
\psi_{t}(\mathbf{r})=\min \left[\left(1+g_{p}\right) \psi_{t-1}(\mathbf{r})-\rho_{t}(\mathbf{r}), \psi_{\max }\right],
$$

where $g_{p}$ is the growth rate for pine trees per year and is set to 0.05 in the present work, and $\min (x, y)=x$ for $x<y$ and $\min (x, y)=y$ otherwise. The tree density in reality cannot grow indefinitely, which is reflected in the form of the condition $\psi_{t}(\mathbf{r}) \leq \psi_{\max }$. When the tree density at a location approaches the upper limit $\psi_{\max }$, 
which is set to 1.0 in the present work, the tree density stops increasing.

\section{Simulation Procedure}

In summary of our model for the spatio-temporal evolution of PNGMs, we implement the growth dynamics for the midge density in Eq. (1) with the egg survival rate in Eq. (2) which depends on climate conditions like temperature and precipitation. The previous generation of midges lay eggs, depending on local tree density, as in Eq. (4), and the growth of trees is affected by the midge density as in Eq. (6). Although our dynamics must be a rough approximation of reality, we have tried to use the known results reported in existing literature. One advantage of our model is that we can try various future climate conditions through the use of the egg survival rate in Eq. (2) which depends on temperature and precipitation.

The time evolution of the midge density, the egg density, and the tree density are governed by the framework presented in Sec. II2. We use two future climate scenarios, RCP4.5 and RCP8.5, for this purpose and download the climate data from Climate Information Portal [34]. The original data have a temporal resolution of one month and a spatial resolution of $1 \mathrm{~km}$, and we make average over time, as explained in Sec. II2 to get the temperature $T_{t}(\mathbf{r})$ and the precipitation $P_{t}(\mathbf{r})$. In model simulations, we use a two-dimensional square lattice with a lattice constant having a linear size $1 \mathrm{~km}$.

We use $\psi_{t=0}(\mathbf{r})=1.0$ as an initial condition for the tree density. Of course, pine trees are not spread uniformly across South Korea, and we have more trees in mountainous areas. Only for simplicity, we use a uniform distribution of the tree density. We choose three harbor cities, Incheon, Mokpo, and Busan, and we assume that the initial PNGM breakout spread starts from there. The reason is that the midge population enters Korea mostly through timber imported from abroad. We assume that all midge eggs hatch to become midge worms, but only part of the worm population becomes adult midges. Accordingly, we use the uniform initial condition $\rho_{t=0}(\mathbf{r})=0$ everywhere, but the initial egg density $\bar{\rho}_{t=0}\left(\mathbf{r}_{i}\right)=0.005$ is assigned at one single lattice point $\mathbf{r}_{i}$ depending on which harbor city is the source position.

Once initial conditions for $\rho, \bar{\rho}$ and $\psi$ are given with fixed all parameter values, we first calculate the evolution of the midge density at each site from Eq. (1). In this procedure, climate conditions and the density of trees are used to calculate the midge density [see Eqs. (2) and (3)]. We then update the density of eggs and the density of trees by using Eq. (44) and Eq. (6), respectively. Adult midges are assumed to put their eggs following Eq. (5). We assume that the initial breakout starts from one of the above mentioned three harbor cities at year 2021 to investigate the spreading pattern in later years till 2050.

\section{RESULTS}

In the 1920's, PNGMs began to spread in Korea. The source locations of spread were harbor cities like Incheon, Mokpo, and Busan, where imported pine timber were unloaded. If timber were infected by PNGM worms, the initial spread of PNGM could start from these harbor cities. We first study how PNGMs would spread in the future if infected timber is imported to one of the harbor cities in Korea. The key ingredient is the future climate conditions, and we use the predictions based on the scenarios RCP4.5 and RCP8.5. We emphasize that our main goal here is to study how future climate changes can alter the pattern of parasite spread in general. Even though our model parameters fit better for PNGM, the model can easily be generalized for other parasite insects.

In our simulations of spread, we assume that the midge spread starts in year 2021 and compute how the midge density evolves in time until the 2050. For simplicity, we pick $t=0$ for the year 2021; thus, 2050 corresponds to $t=29$. For the initial condition of midge density, we use $\rho_{0}(\mathbf{r})=0.0$ for all locations; i.e., no adult midges exist at $t=0$. For the initial values of the egg density, we set $\bar{\rho}_{0}(\mathbf{r})=0.005$ for one lattice point in the harbor city area where the initial spread occurs. Of course, any location ,except for this source lattice point, is assigned $\bar{\rho}_{0}(\mathbf{r})=0$. For the location of the source of midge spread, we pick three cities, Incheon, Mokpo, and Busan based on what happened in 1920's. We then simulate our model for all six $(=3 \times 2)$ different cases, i.e., three source locations and two climate conditions.

The spread of midges is shown to be not so fast and even after almost 30 years from the initial breakout of spread, midges are found not to have spread across the country as shown in Fig. 11where we display midge densities in (a) and (c) together with tree densities in (b) and (d). For simplicity, we plot the results from three different breakout locations (Incheon, Mokpo, and Busan) altogether in one Korean map for a given future climate scenario RCP4.5 for (a) and (b), and RCP8.5 for (c) and (d). As is clearly seen, the midge density propagates in space like a wavefront, and three density waves starting from three locations do not meet yet. Our model dictates how midges tend to migrate to sites where climate conditions are better and where more trees exist. The midge density propagates to locations far from the initial breakout site as time goes on. Infected pine trees tend to die [see Eq. (6)] and it takes a long time for pine trees to recover their initial level of tree density. Consequently, the locations where midge densities are high tend to form a circle-like structure, the radius of which expands in time. When pine trees die of PNGM infection, PNGMs can hardly flourish later because of the lack of the trees in which PNGM worms can survive. We thus expect PNGM density eventually to spread from the initial source to the whole country after which they become extinct. Without PNGMs, pine trees grow back and approach a suitable level of tree density. The circular shape of the wave- 


\section{RCP4.5}

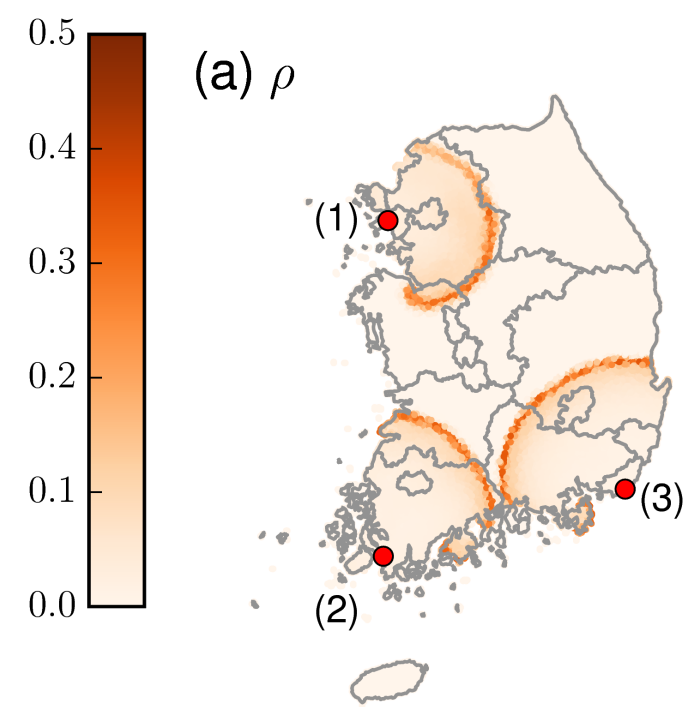

(b) $\psi$
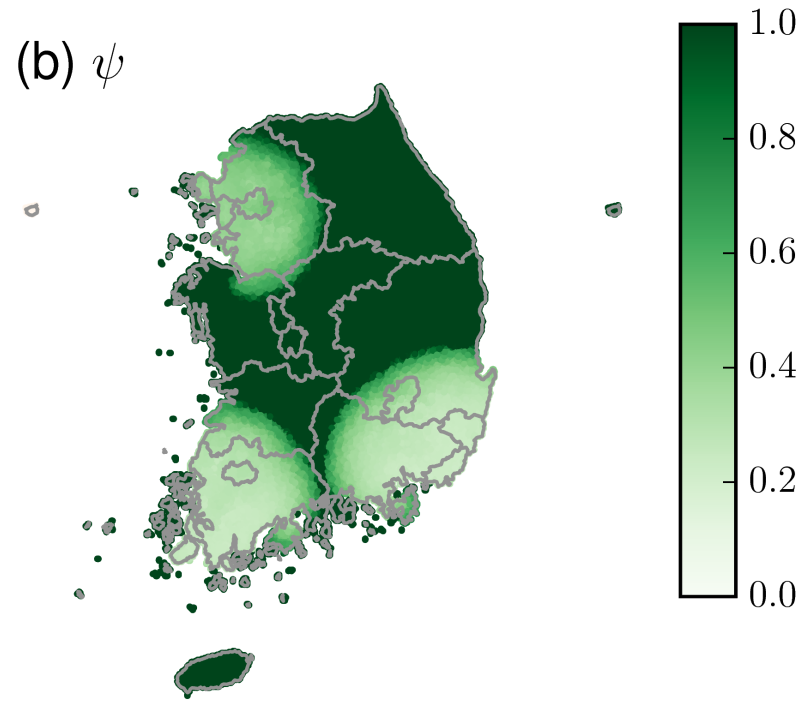

\section{RCP8.5}

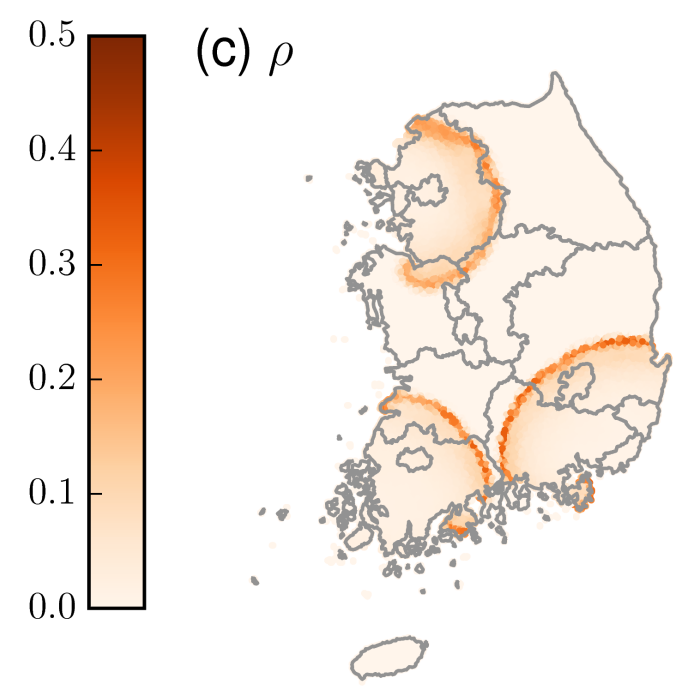

(d) $\psi$

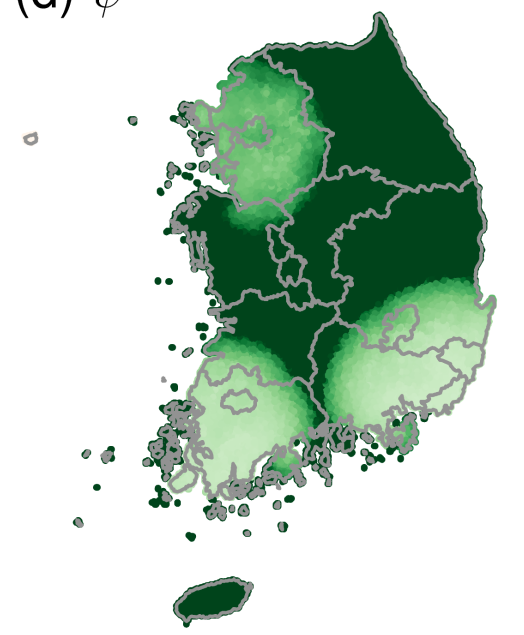

1.0

0.8

0.6

0.4

0.2

FIG. 1. The densities of PNGM $(\rho)$ and trees $(\psi)$ in 2050 based on two climate conditions: RCP4.5 for (a) $\rho$ and (b) $\psi$, and RCP8.5 for (c) $\rho$ and (d) $\psi$, respectively. Initial breakout of PNGM is assumed to occur in a harbor city [(1) Incheon, (2) Mokpo, or (3) Busan as shown in (a)] in 2021. Since spread of midges starting from one of three harbor cities is still spatially limited in 2050, we plot three different results for three different source locations in one Korean map for convenience, both for midge density and for tree density. The results from RCP4.5 and RCP8.5 are not much different from each other, but the spread starting from Incheon is shown to be a little faster in RCP8.5 than in RCP4.5. We note that the density of trees in the region where PNGM has already killed most trees remains small, but nonzero, in coming years.

front of midge density originates from the isotropy in the model. However, in reality, the pine trees do not grow everywhere, and there are regions where pine trees grow better or worse exist. We believe that our model has room for further improvement by making the maximum tree density $\psi_{\max }$ depend on $\mathbf{r}$.

We define the spread radius $R_{i}(t)$ for the midge density when the spread has started from the source city $i$ at position $\mathbf{r}_{i}$ as

$$
R_{i}(t) \equiv \sum_{\mathbf{r}}\left|\mathbf{r}-\mathbf{r}_{i}\right| \rho_{t}(\mathbf{r}) .
$$

For convenience, we choose $\mathbf{r}_{i}$ as one lattice point in the harbor city $i \in\{$ Incheon, Mokpo, Busan\}, where the 

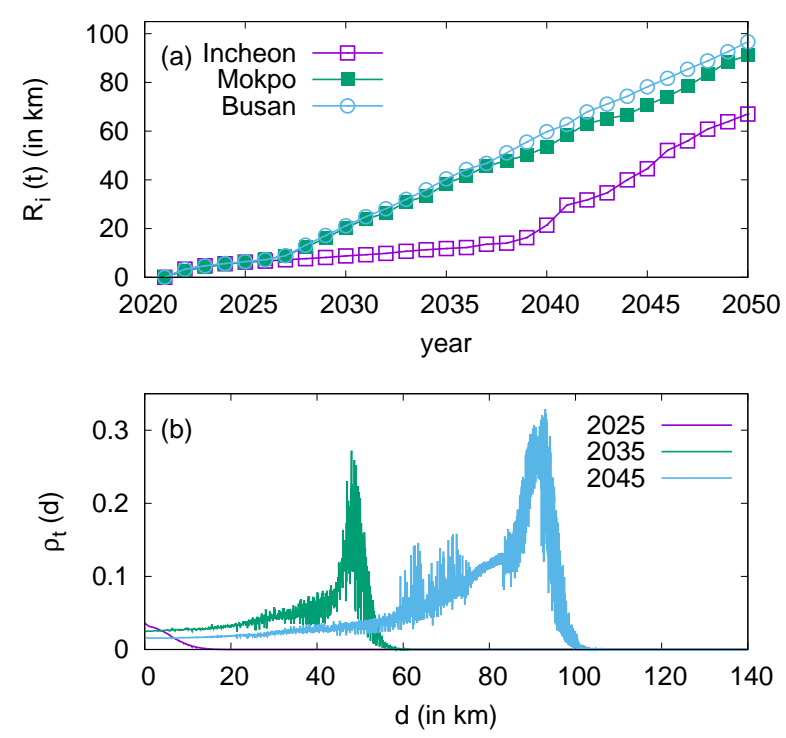

FIG. 2. (a) The radius of spread in Eq. (7) increases as time proceeds. Three harbor cities, Incheon, Mokpo, and Busan, are used as the initial breakout sites of the PNGM spread, and the climate condition is based on the RCP4.5 scenario. After an early stage in which the radius increases very slowly, the radius kicks off beyond the outbreak points which depend on source cities. The radii of spread for Mokpo and Busan show sudden changes in the spreading speed at around 2026, but Incheon shows a much later outbreak point of 2038. (b) The midge density profile at different times, 2025, 2035, and 2045, for RCP4.5-based climate condition. The distance $d$ from the source position with Busan as the source city is used for the horizontal axis.

initial condition $\bar{\rho}_{0}\left(\mathbf{r}_{i}\right)=0.005$ has been assigned. At $t=0, \rho_{0}(\mathbf{r})$ is localized to this source location; thus, $R_{i}(t=0)=0$. As time evolves, $\rho_{t}(\mathbf{r})$ extends to cover a broader region; thus, $R_{i}(t)$ increases.

Figure 2(a) displays the temporal change of $R_{i}(t)$ for each source city when the climate scenario RCP4.5 is used. In the initial stage of PNGM spread, the radius first increases very slowly, and then suddenly kicks off after some years. The radius increases at a rate of $1 \mathrm{~km} /$ year for first five to six years for Mokpo and Busan as a source locations. Similar stagnation is observed also for Incheon as source location, but it lasts for a much longer time until around the year 2038 with the slower increasing rate of $0.6 \mathrm{~km} /$ year for the spread radius. Interestingly a similar stagnation behavior has been observed in reality for the past spread pattern of PNGMs [35]. In Ref. 15, the radius of spread was shown to kick off after a stagnation period, and the outbreak point coincides well with the instant when the population approaches the carrying capacity. When the midge density is small, the damage from parasites is soon repaired, which allows the midge to lay eggs uniformly around its current position. In this case, the spatial midge density exhibits a unimodal shape with the center at the source location. As the midge population grows further, the ruined tree density cannot recover in a short time, but keeps decreasing gradually due to parasitizing midges. In such case of high midge density, the diffusion of midges exhibits a bias toward an outgoing direction where more trees still exist. Consequently, the location of the maximum midge density drifts away from the original source location, and the radius of spread increases faster.

When the midge spread starts from Incheon, the initial stage of stagnation is found to be longer than it is for the other source cites Mokpo and Busan. The difference appears to originate from the different climate conditions, the precipitation from March to May in particular. We observe that according to the RCP4.5-based prediction, precipitation around Incheon gradually increases after year 2035 and thus $G(P)$ in Eq. (3) takes the almost maximum value of unity at years 2039 and 2040 . This then leads to an increase in the midge density, which soon reduces the tree density. If the tree density becomes smaller around the Incheon area, the midges then migrate outward, and the radius in Fig. 22(a) kicks off at around year 2040. After the early stage of migration, the locations where the midge densities are larger begin to move out from the source position. When this happens, the speed of diffusion is observed to become faster. The rate of increase of the radius for Mokpo and Busan as source cites after the kickoff occurs are about $3.5 \mathrm{~km} /$ year and $3.8 \mathrm{~km} /$ year, respectively, while the corresponding value for Incheon is $4.7 \mathrm{~km} /$ year.

In Fig. 2(b), the density of midges with Busan as the source city is shown as a function of the distance from Busan at three different instants, 2025, 2035, and 2045. In the early stage of diffusion at year 2025, the density shows a Gaussian-like shape with a maximum at the position of the source city (at null distance) as expected. After the early stage of diffusion, the Gaussian-like shape with its maximum at the origin begins to change, and the maximum shifts away from the origin. This is due to the decrease in the tree density near the origin, which drives midges away in an outward direction, as explained above. Of course, if midges move away from the origin, the tree density near the source location can recover. However, the midges do not come back to the origin because they have to overcome the harsh region in which the tree density is lower.

We then investigate how different RCP scenarios affect the prediction of midge density in the future. We use Incheon as initial source position and compare the radius of spread obtained from RCP4.5 and RCP8.5 in Fig. 3. For RCP4.5, the radius kicks off at around 2039 while it kicks off at around 2031 for RCP8.5. As explained above, the sudden change in the slope in Fig. 3 at these outbreak points originates from the competition between two growth rates, that for trees and that for midges. The difference in the outbreak points of RCP4.5 and RCP8.5 can be explained as follows: Precipitation significantly increases in the northern part of South Korea as the greater greenhouse gas emission is assumed. Increased precipitation yields the increase of the midge population 


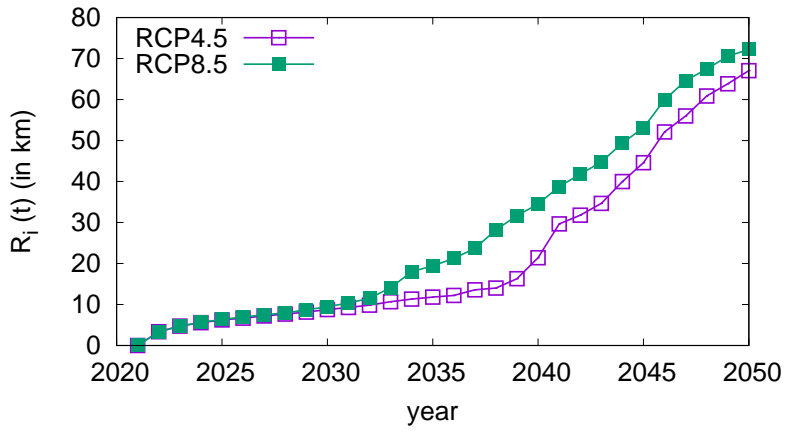

FIG. 3. The radius of spread $R_{i}(t)$ in Eq. (7) for Incheon as the source city with climate conditions based on RCP4.5 and RCP8.5. A sudden increase in the radius of spread occurs earlier for RCP8.5 than for RCP4.5, which can be explained by the sufficiently large value of precipitation in RCP8.5 (see text for details).

[see Eqs. (2) and (3)], which shifts the outbreak point to an earlier time. We recognize that the difference of such outbreak points between RCP4.5 and RCP8.5 is almost indiscernible for other source cities, Mokpo and Busan. It suggests that the climate conditions in southern cities are already suitable for the fast growth of the midge density.

\section{CONCLUSION}

We have proposed a spatio-temporal spread model of an insect species like the pine needle gall midge (PNGM) that parasitizes trees. The model includes the density of adult midges, the density of midge eggs, and the density of pine trees as dynamic variables and describes how their dynamics are coupled to each other. One of the main research goals of the present paper has been to investigate how future climate conditions can alter the spread pattern of insects. For this purpose, we have used climate predictions based on two standard scenarios, RCP4.5 and RCP8.5, with different future estimates of greenhouse gas emission. We have downloaded future climate data for each scenario which contains grid data for temperature and precipitation with a temporal resolution of one month. The density of PNGMs is calculated by the growth equation similar to the logistic equation, in which we use the climate-dependent survival rate. The adult midges are modeled to lay eggs near their current positions in distance of $5 \mathrm{~km}$, and the density of eggs depends on the local tree density.

From our extensive simulations, we observed that the radius of spread as a function of time has different increase rates in the early and the late stages of diffusion: In the early stage in which the midge density is still small the diffusion of midges is slow whereas after the outbreak point of spread the diffusion becomes much faster. We emphasize that a similar result of a change in diffusion speed has been found in the field research [35]. For a variety of different pests including PNGM investigated in the present paper, the survival rate of parasites is greatly influenced by the climate condition: More midges survive to become adults when both precipitation and temperature are sufficiently high. We have observed in our simulations that when Mokpo and Busan (located along the southern cost of South Korea) are used as the source sites of spread, the outbreak point occurs at a much earlier time than it does when Incheon (located in the midwestern coast of Korean peninsula) is the source site. We have investigated the reason for the difference between northern and southern source sites and have found that it originates from the different climate conditions, precipitation in particular. Southern regions of South Korea have sufficiently high precipitation for midge population to grow fast, which then significantly reduces the tree density near the source site. If this happens, midges tend to migrate to a region with a high tree density and thus spread faster in a radially outward direction from the source site. Consequently, the outbreak point for the faster diffusion occurs in an early stage if the precipitation near the source site is sufficiently high.

As the greenhouse gas emission is increased, the RCP scenario changes from RCP4.5 to RCP8.5, and climate variables such as temperature and precipitation in the future are greatly influenced. Two different climate conditions, RCP4.5 and RCP8.5, have been found to result in almost the same spread pattern with a hardly recognizable change of the outbreak points for Mokpo and Busan as source cities. In contrast, RCP8.5 yields a much earlier outbreak point than RCP4.5 for Incheon. We interpret that this difference in diffusion behavior between southern and northern source cites in South Korea should originate from the difference in the precipitation in the two regions. In the southern part of South Korea, both RCP4.5 and RCP8.5 predict sufficiently high precipitation and the spread kicks off early. However, for the northern part of South Korea, the precipitation predicted by RCP 8.5 is higher than that predicted by RCP 4.5 , leading to a difference in the outbreak point for the case of Incheon as the source site. In more detail, an average precipitation higher than $50 \mathrm{~mm}$ has been observed to induce an increase in the midge density in successive $2 \sim 3$ years, which reduces the host tree density; then, midges spread in an outward direction faster seeking regions with a high tree density. Such a condition of high precipitation near Incheon has been found to be well satisfied at early times in RCP8.5, but not in RCP4.5, leading to a shift in the outbreak point to an early time for RCP8.5.

We believe that our spatio-temporal growth model for PNGM spread can easily be generalized for similar parasite insects. The present model can also be generalized to mimic the spreading of parasites through road traffic. For the case when infected timber is moved to other cities through ground transportation, changing our model equation to cover such a long-distance spread is straightforward. 


\section{ACKNOWLEDGMENT}

This study was carried out with the support of the Research Program of Rural Development Administration, Republic of Korea (Project No. PJ01156304)

\section{REFERENCES}

[1] B. Meerson and P. V. Sasorov, Phys. Rev. E 83, 011129 (2011).

[2] M. G. Clerc, D. Escaff, and V. M. Kenkre, Phys. Rev. E 72, 056217 (2005).

[3] C. Escudero, J. Buceta, F. J. de la Rubia, and K. Lindenberg, Phys. Rev. E 69, 021908 (2004).

[4] J. D. Murray, Mathematical Biology, (Springer, Berlin, 1993).

[5] A. Tsoularis and J. Wallace, Math. Biosci. 179, 21 (2002).

[6] E. E. Holmes, M. A. Lewis, J. E. Banks, and R. R. Veit, Ecology 75, 17 (1994).

[7] H. Caswell and R. Etter, B. Math. Biol. 61, 625 (1999).

[8] Y. Harada and Y. Iwasa, Res. Popul. Ecol. 36, 237 (1994).

[9] D. E. Hiebeler and B. R. Morin, J. Theor. Biol. 246, 136 (2007).

[10] M. Ikegamia, D. F. Whighamb, and M. J. A. Wergera, Ecol. Model. 234, 51 (2012).

[11] C. J. Rhodes and R. M. Anderson, J. Theor. Biol. 180, 125 (1996).

[12] H. Xuan, L. Xu, and L. Li, Ann. Oper. Res. 168, 81 (2009).

[13] T.-S. Chon, S. D. Lee, and B.-Y. Lee, New Phys.: Sae Mulli 38, 184 (1998).

[14] M. A. Brockhurst, A. Buckling, V. Poullain, and M. E. Hochberg, Evolution 61, 1238 (2006).

[15] S. D. Lee, S. Park, Y.-S. Park, Y.-J. Chung, B.-Y. Lee, and T.-S. Chon, Ecol. Model. 203, 157 (2007).

[16] G. Szabó and C. Tőke, Phys. Rev. E 58, 69 (1998).

[17] P.-P. Li, J. Kea, L.-L. Jiang, X.-Z. Yuan, and Z. Lin, Eur. Phys. J. B 86, 168 (2013).

[18] J.-H. Cho and S.-H. Lee, J. Korean Phys. Soc. 64, 746 (2014).

[19] K. N. Park and J. S. Hyun, J. Korean For. Soc. 61, 20 (1983).

[20] Y. Son, J.-H. Lee, and Y.-J. Chung, J. Appl. Entomol. 131, 674 (2007).

[21] B. Y. Lee, T. Miura, and Y. Hirashima, ESAKJA 23, 119 (1985).

[22] T.-S. Chon, Y.-S. Park, J.-M. Kim, B.-Y. Lee, Y.-J. Chung, and Y. Kim, Environ. Entomol. 29(6), 1208
(2000)

[23] Y.-S. Park and Y.-J. Chung, Forest Ecol. Manag. 222, $222(2006)$.

[24] K.-W. Ahn, H.-S. Lee, D.-C. Seo, and S.-H. Shin, J. Korean Soc. Geosp. Inf. Syst. 6, 105 (1998) (in Korean).

[25] IPCC IS92 Scenarios http://sedac.ipcc-data.org/ddc/is92/

[26] N. Nakićenović, J. Alcamo, G. Davis, et al., in Special Report on Emissions Scenarios: A special report of Working Group III of the Intergovernmental Panel on Climate Change, edited by N. Nakićenović and R. Swart, (Cambridge University Press, Cambridge, 2000).

[27] M. Collins, R. Knutti, J. Arblaster, J.-L. Dufresne, T. Fichefet, P. Friedlingstein, X. Gao, W. J. Gutowski, T. Johns, G. Krinner, M. Shongwe, C. Tebaldi, A. J. Weaver, and M. Wehner, in Climate Change 2013: The Physical Science Basis. Contribution of Working Group I to the Fifth Assessment Report of the Intergovernmental Panel on Climate Change, edited by T. F. Stocker, D. Qin, G.-K. Plattner, M. Tignor, S. K. Allen, J. Boschung, A. Nauels, Y. Xia, V. Bex, and P. M. Midgley, (Cambridge University Press, Cambridge, 2013), Chap. 12.

[28] F. Giorgi, E. Coppola, F. Solmon, L. Mariotti, et al., Clim. Res. 52, 7 (2012).

[29] H.-S. Kang, D.-H. Cha, and D.-K. Lee, J. Geophys. Res. 110, D10105 (2005).

[30] H.-M. H. Juang, S.-Y. Hong, and M. Kanamitsu, Bull. Amer. Meteor. Soc. 78, 2125 (1997).

[31] W.C. Skamarock, J. B. Klemp, J. Dudhia, D. O. Gill, D. M. Barker, W. Wang, and J. G. Powers, A description of the Advanced Research WRF version 2, (National Center for Atmospheric Research, Boulder, 2005).

[32] T. Davies, M. J. P. Cullen, A. J. Malcolm, M. H. Mawson, A. Staniforth, A. A. White, and N. Wood, Q. J. R. Meteorol. Soc. 131,1759 (2005); G. M. Martin, M. A. Ringer, V. D. Pope, A. Jones, C. Dearden, and T. J. Hinton, J. Climate 19, 1274 (2006).

[33] M.-S. Suh, S.-G. Oh, D.-K. Lee, D.-H. Cha, S.-J. Choi, C.-S. Jin, and S.-Y. Hong, J. Climate. 25, 7067 (2012).

[34] Climate Information Portal (Korean) http://www.climate.go.kr/

[35] S. H. Park, Master thesis, Pusan National University, 2000. 